\title{
Three-dimensional structure of the large ribosomal subunit from Escherichia coli
}

\author{
M.Radermacher, T.Wagenknecht, A.Verschoor \\ and J.Frank \\ Wadsworth Center for Laboratories and Research, New York State \\ Department of Health, Albany, NY 12201, USA \\ Communicated by R.Henderson
}

The three-dimensional structure of the large $(50 S)$ ribosomal subunit from Escherichia coli has been determined from electron micrographs of negatively stained specimens. A new method of three-dimensional reconstruction was used which combines many images of individual subunits recorded at a single high tilt angle. A prominent feature of the reconstruction is a large groove on the side of the subunit that interacts with the small ribosomal subunit. This feature is probably of functional significance as it includes the regions where the peptidyl transferase site and the binding locations of the elongation factors have been mapped previously by immunoelectron microscopy.

Key words: electron microscopy/image processing/ribosome structure/three-dimensional reconstruction

\section{Introduction}

Knowledge about the three-dimensional structure of the ribosome and its component subunits is severely limited due to the inherent complexity of this organelle. A number of models have been proposed for the large (50S) and small (30S) subunits of the procaryotic ribosome based upon visual examination of electron micrographs along with correlative information from other physical and chemical techniques (reviewed by Wittman, 1983). These models are necessarily limited in resolution (probably no better than $5 \mathrm{~nm}$ ) and disagree in detail with one another even at this level.

The unique problems associated with a high-resolution structural determination by $\mathrm{X}$-ray crystallographic methods for a structure as complex as the ribosome have been discussed (Liljas, 1982; Yonath, 1984), and it will no doubt be many years before the ribosomal structure at high resolution is known. Quantitative three-dimensional reconstruction applied to electron micrographs of ribosomes or the component subunits promises to provide the first reliable structural models for the ribosome at moderate resolution $(2-3 \mathrm{~nm})$. Recently, there have appeared several preliminary accounts of three-dimensional reconstructions of the procaryotic 50S subunit from two-dimensional crystals (Arad et al. 1984; Lake, 1985). However, thus far, these studies have not produced a three-dimensional model to supersede the visually derived models. Another group has computed threedimensional reconstructions of individual ribosomal subunits from series of micrographs obtained by incrementally tilting the specimen (Hoppe, 1982, 1983; Oettl et al., 1983; Hegerl et al., 1984). However, the method that they used suffers from several disadvantages. First, since many micrographs are recorded of each subunit to be reconstructed, the accumulated electron dose is sufficient to damage the specimen substantially. Second, it requires an extreme amount of effort to improve the signal-to-noise ratio of the three-dimensional map by averaging over many individual reconstructions.

Here we describe a three-dimensional structure for the $50 \mathrm{~S}$ ribosomal subunit from Escherichia coli obtained by a new method of reconstruction, applicable to electron microscopic images of single macromolecules that exhibit a well defined orientation with respect to the specimen grid (Radermacher et al. , 1986a, b; 1987). A single image of such a specimen tilted by a fixed angle $\theta$ of $\geq 45^{\circ}$ is sufficient to provide all the data needed for a three-dimensional reconstruction (Frank et al., 1978). Because the particles lie parallel to the specimen plane but have random in-plane orientations, $\phi_{i}$, the projections of $N$ particles visible in a micrograph of the tilted specimen provide a complete conical tilt series with projections at angles $\left(\theta, \phi_{i}\right), i=1 \ldots N$. Since only a single exposure is needed the method is suitable for specimens sensitive to radiation damage. The azimuthal angles in the conical tilt series are determined from a $0^{\circ}$ micrograph of the same specimen area. As this latter micrograph serves only for the determination of the angles $\phi_{i}$ but is not used as input data to the three-dimensional reconstruction, this second exposure does not contribute to the radiation damage of the resulting structure. Averaging, also causing an increase in signalto-noise ratio of the result, is intrinsically achieved in this method of reconstruction in two ways. First the minimum number of projections required to achieve a certain resolution already entails a substantial oversampling of the low spatial frequency range of the Fourier domain, resulting in effective (albeit frequencydependent) averaging. Secondly the number of projections we have used is much larger than the minimum number (489 as compared to 51) that are required to achieve this resolution from a conical tilt series with equal angular increments, so that even the highest spatial frequency components are multiply measured, and noise in the lower spatial frequency range is further reduced.

The three-dimensional model that we have obtained is the most detailed yet described for the $50 \mathrm{~S}$ ribosomal subunit, and several new structural features have been resolved, most notably a deep groove near the top of the subunit in a region believed to contain the peptidyl transferase center and binding sites for the elongation factors.

\section{Results}

\section{Electron microscopy and two-dimensional image analysis}

Electron micrographs of the same field of negatively stained ribosomal subunits taken at tilt angles of $50^{\circ}$ and $0^{\circ}$ are shown in Figure $1 \mathrm{~A}$ and $\mathrm{B}$ respectively. From the micrographs of the untilted specimen it can be seen that most $(>75 \%)$ of the subunits lie in a preferred orientation termed the 'crown' view (Tischendorf et al., 1974). Images showing the crown orientation were selected from five tilted/untilted pairs of micrographs for inclusion in the three-dimensional reconstruction algorithm.

The subunits were prepared for microscopy by a sandwich 

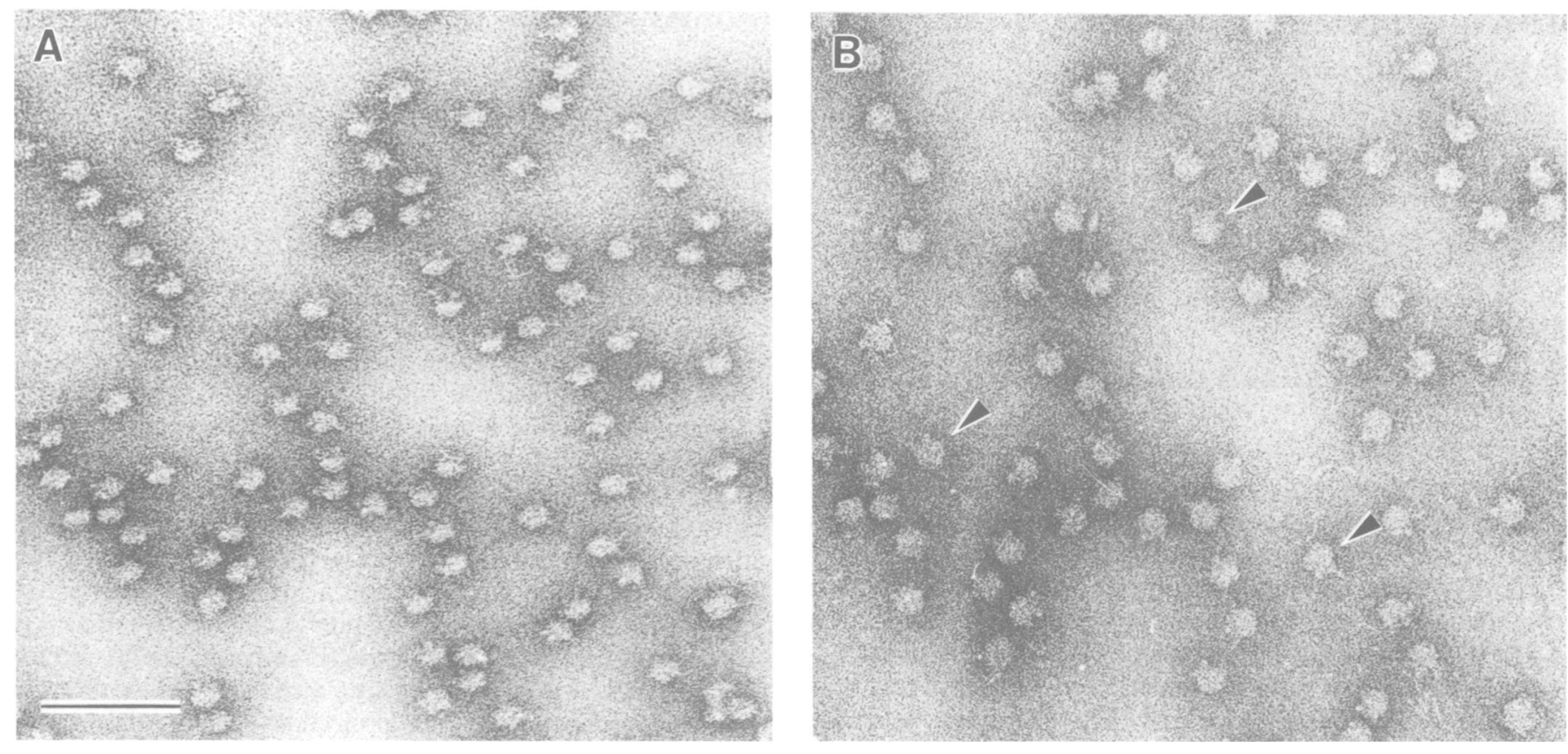

Fig. 1. Electron micrographs of negatively stained $50 \mathrm{~S}$ ribosomal subunits from $E$. coli recorded at tilt angles of $50^{\circ}(\mathbf{A})$ and $0^{\circ}$ (B). Several subunits in 'crown' orientation are indicated in (B). Bar represents $100 \mathrm{~nm}$.

technique (Tischendorf et al., 1974; Stöffler and StöfflerMeilicke, 1983) in which the specimen is bounded above and below by carbon films. We have found that this method of specimen preparation gives a uniformity and reproducibility of staining that is difficult to achieve by more conventional procedures. This can be seen by comparing independent averages of crown views (untilted) obtained after rotational and translational alignment of the individual images by correlation methods (Frank et al., 1978, 1985). Two-dimensional averages from two of the $0^{\circ}$ micrographs of the particles that we used for the threedimensional reconstruction show striking reproducibility (Figure $2 \mathrm{~A}$ and $\mathrm{B}$ ); essentially all of the detailed features are present in both averages. A phase residual analysis (Frank et al., 1981), a quantitative test of the similarity of the two $0^{\circ}$ averages, established that the reproducibility extended to $2.9 \mathrm{~nm}$; another measure, the ring correlation test (Saxton and Baumeister, 1982; van Heel et al., 1982) gave a value of $2 \mathrm{~nm}$. A similar degree of reproducibility was found for all five pairs of micrographs chosen for the analysis. We also determined two independent $0^{\circ}$ averages from the entire set of particles used in the threedimensional reconstruction. The reproducibility for these averages extended to $2.2 \mathrm{~nm}$ by the phase residual criterion and $1.6 \mathrm{~nm}$ by the ring correlation test (Figure $2 \mathrm{C}$ and $\mathrm{D}$ ); these values set an upper limit for the resolution that could be expected for the three-dimensional reconstruction.

\section{Three-dimensional reconstruction}

A new method of three-dimensional reconstruction was used (Radermacher et al., 1986a,b; 1987) in which many images of tilted, individual 50S subunits of the crown type were combined to yield a single three-dimensional reconstruction. The reconstruction was computed using 489 images of 50S ribosomal subunits in the crown orientation selected from micrographs of the specimen tilted by $50^{\circ}$. The corresponding images from the untilted specimen are used only to determine the azimuthal angle of each particle with respect to an arbitrarily chosen reference
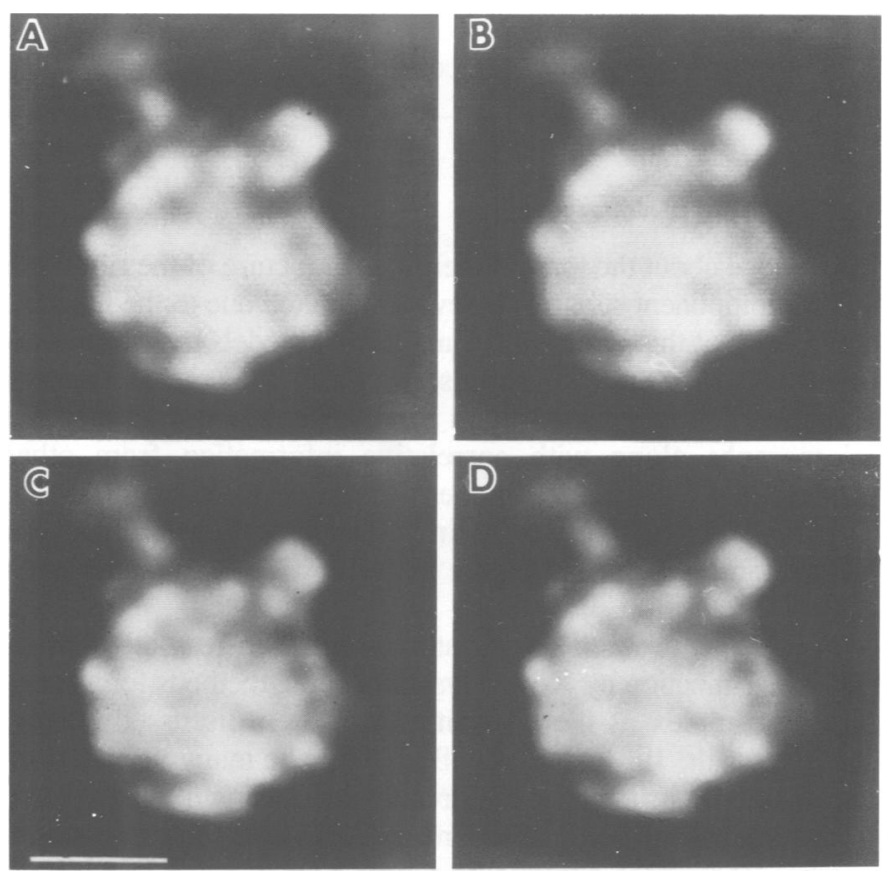

Fig. 2. Averages of images in the crown orientation (untilted). (A), (B) Independent averages of aligned images of the 'crown' type obtained from two of the micrographs used to compute the three-dimensional reconstruction. (C) Average of 245 of the 490 images used in the reconstruction. (D) Average of all 490 images. Bar represents $10 \mathrm{~nm}$.

orientation, and for assessing the reproducibility of the images from different micrographs as described above. They were not used as input projections to the three-dimensional reconstruction, and thus the electron dose used for the image of the untilted specimen did not contribute to the accumulated electron dose.

The reconstruction has been low-pass filtered to a limiting resolution of $3 \mathrm{~nm}$ (see Materials and methods), and the resulting 


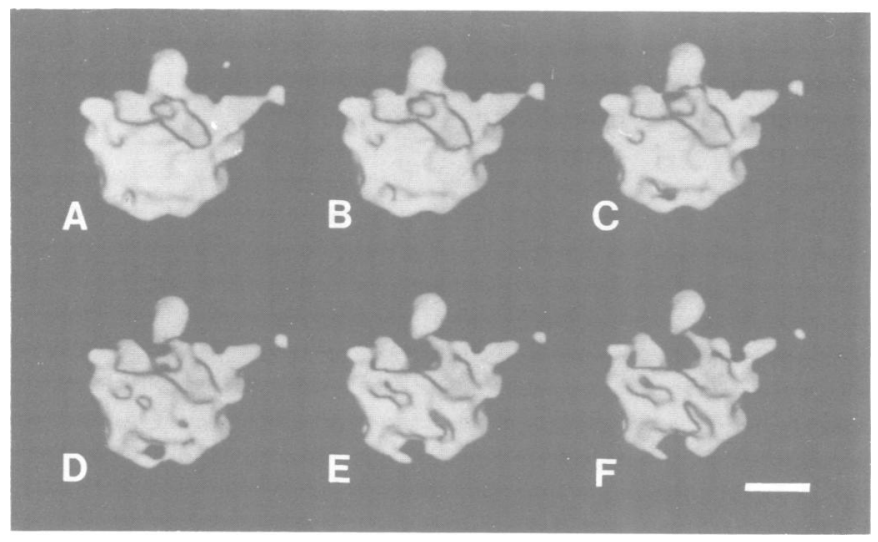

Fig. 3. Surface representations of the three-dimensional reconstruction of the $50 \mathrm{~S}$ ribosomal subunit. Decreasing density thresholds were used. The enclosed volumes of the models shown are: A $2340 \mathrm{~nm}^{3}$, B $2100 \mathrm{~nm}^{3}$, C $1860 \mathrm{~nm}^{3}$ D $1620 \mathrm{~nm}^{3}$, E $1370 \mathrm{~nm}^{3}$, F $1130 \mathrm{~nm}^{3}$.

three-dimensional structure was visualized by a series of computer-generated surface representations calculated for increasing threshold levels (Figure 3). The density levels chosen for computing the surfaces (Figure $3 \mathrm{~A}, \mathrm{~B}$ and $\mathrm{C}$ ) were in the range where the density gradient was greatest in the vicinity of the particle boundary.

The volume of the model (Figure 3A) corresponds to $2340 \mathrm{~nm}^{3}$, in good agreement with the values of 2500 and $2600 \mathrm{~nm}^{3}$ determined for the hydrated particle by small-angle $\mathrm{X}$-ray scattering (Tardieu and Vachette, 1982; Meisenberger et al., 1984). Good agreement is also found for the overall dimensions of the model with values estimated from small-angle $\mathrm{X}$ ray scattering and electron microscopy (summarized by Wittman, 1983). It is particularly noteworthy that the maximal dimension of the model in the viewing direction perpendicular to the specimen plane (i.e. perpendicular to the crown view, Figure 4C) corresponds to $14 \mathrm{~nm}$ as compared to the values of 13.5 - $16 \mathrm{~nm}$ obtained by Tardieu and Vachette (1982), indicating that little, if any, flattening or collapse of the specimen occurred during the preparation for electron microscopy. Unlike some algorithms for computing three-dimensional reconstructions of symmetric particles in which a cylindrical or spherical shape is assumed, no implicit assumptions are made regarding the outer shape of the particle in the reconstruction scheme we used. In other words, if the subunits were flattened, then the computed three-dimensional reconstruction would also appear flattened.

In order to aid interpretation of the reconstruction, solid models were constructed for the models shown in Figure $3 \mathrm{~B}$ and $\mathrm{E}$ (Figures 4 and 5 respectively). The three-dimensional model (Figure 4) shows all of the major surface substructures present in the visually derived models: the central protuberance, L7/L12 stalk, $\mathrm{Ll}$ ridge, and the roughly hemispherical main body. One of the most striking features of the reconstruction is a deep groove (Figure 4A) lying just below the central protuberance and on the face of the subunit that binds the $30 \mathrm{~S}$ subunit in the $70 \mathrm{~S}$ monosome. This feature, which we will refer to as the interface canyon', is $\sim 16 \mathrm{~nm}$ in contour length and extends from the base of the stalk, where it is widest and deepest $(\sim 4 \times 4 \mathrm{~nm})$, across the subunit to the L1 ridge where it is open from above. A continuous shelf, sloping downwards on the stalk side in Figure 4A, forms the lower edge of the canyon. The floor of the canyon is not smooth and appears as three subregions or pockets. The deep central pocket (labeled P2 in Figure 4A), located directly below the head, has a hole at its base leading to the back of the subunit. Two additional, smaller holes are present in the canyon between the central protuberance and the L7/L12 stalk; one is located in the pocket labeled P1 and the other is on the upper wall of the canyon just below and on the left side of the central protuberance. The stain-excluding regions forming the borders of the latter hole along with those from the hole in P1 comprise three bridges of density connecting the central protuberance to the body of the subunit (Figure 4B). If the 23S rRNA extends into the central protuberance as suggested by Noller and Lake (1984), then one of these bridges might correspond to the $23 \mathrm{~S}$ rRNA entering and/or leaving this region. A fourth, smaller pocket (P4) of stain inclusion $(\sim 2 \mathrm{~nm}$ wide by $1-2 \mathrm{~nm}$ deep) is present just in front of the L1 ridge and could be considered as an extension of the interface canyon.

Below the interface canyon is a plateau region devoid of any prominent surface features (Figure 4A and C). The visually derived models generally show a much larger plateau region or a concavity in this region (Lake, 1976, 1985; Vasiliev et al., 1983; Stöffler and Stöffler-Meilicke, 1984).

On the outside margin of the subunit, directly below the L7/L12 stalk, is a well-defined ridge feature (' $R$ ' in Figure 4) with a frontto-back extent of $>5 \mathrm{~nm}$. It is in the vicinity of the ' $\mathrm{L} 11$ domain' identified by Stöffler-Meilicke et al. (1983) and appears also to be present in reconstructions of stalkless particles (Hoppe, 1982; Hegerl et al., 1984).

At the bottom of the subunit there is an elongated depression similar to a feature incorporated into one of the visually derived models (Vasiliev et al., 1983) and also visible in averaged images of subunits in the crown view (Kiselev et al., 1983; Figure 2 of Verschoor et al., 1985) although its location and shape in three dimensions were of course not apparent until now. This feature is more clearly visible from the back of the subunit (Figure $4 B$, labeled ' $N$ '). Approximately $3 \mathrm{~nm}$ from the notch on the interface side is a smaller depression (labeled $N^{\prime}$ ); if a lower density level is chosen to represent the surface the major and minor notches merge (Figure 5A).

The back of the subunit is convex in overall shape (Figure 4C) and does not show significant substructure except for a knob of stain exclusion located between the head and L1 ridge ('backknob', labeled 'BK' in Figure 4B) and the 'notch' feature mentioned above.

A general problem in determining the surface topography of negatively stained specimens is that often the surface features observed depend upon the density threshold chosen to represent the surface. All of the features described thus far, for the model in Figure 4, are not especially sensitive to the particular density level used. However, with the density level chosen to generate the surface shown in Figures $3 \mathrm{E}$ and 5, some additional features are observed, including four cavities [C1 on the interface side (Figure 5A); $\mathrm{C} 2-\mathrm{C} 4$ on the back (Figure $5 \mathrm{~B}$ )] and a groove on the back (labeled 'GR' in Figure 5B). The groove extends from the open region below the central pocket, $\mathrm{P} 2$, downwards about half way to the bottom of the subunit.

An interesting characteristic of the reconstructed subunit is that it shows distinct density variations throughout its volume. This can be appreciated from the sections through the volume shown in Figure 6. For example, a central section (Figure 6B) shows the greatest stain exclusion in defined subregions near the perimeter of the subunit, whereas much of the interior region is, on average, higher in density (i.e. showing less stain exclusion). 

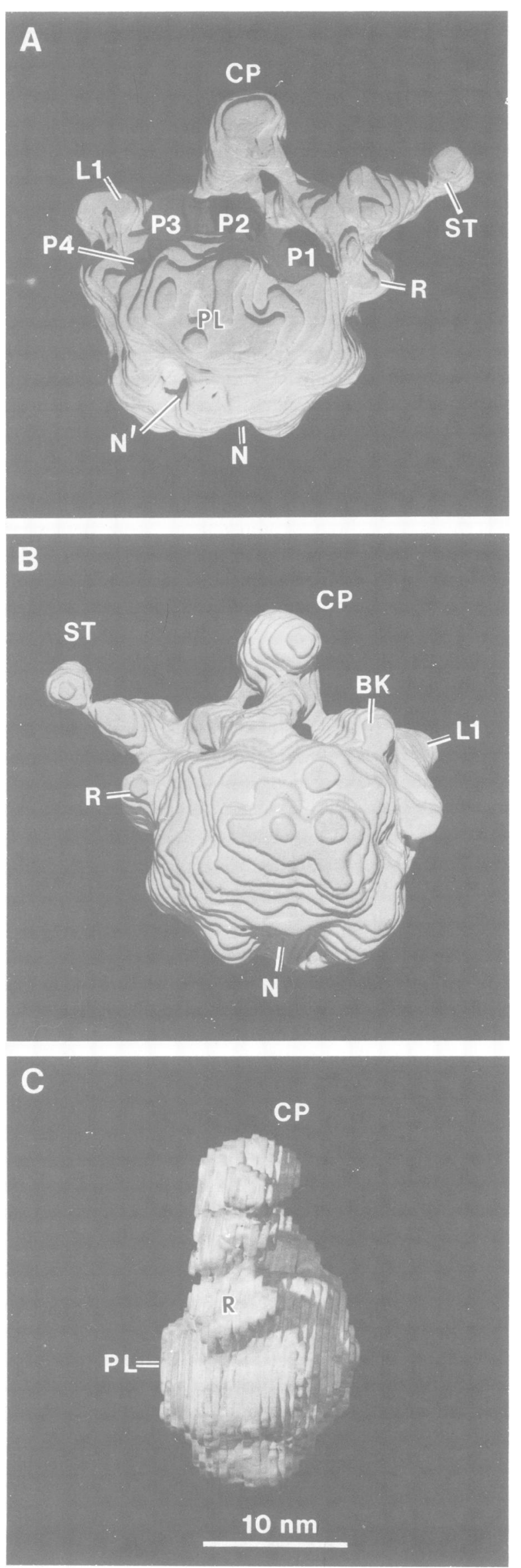

\section{Discussion}

\section{Comparison with other three-dimensional models}

It is instructive to compare the three-dimensional model we have obtained for the 50S ribosomal subunit with models that have been proposed based upon direct, visual interpretation of electron micrographs. At least five models have been proposed (reviewed by Wittman, 1983) which differ in the arrangement of the three protuberances and in the overall shape of the main body. Our model resembles the visually derived models of Lake (1976), Stöffler and Stöffler-Meilicke (1984), and Vasiliev et al. (1983) in these respects, but beyond these gross features, our reconstruction reveals many heretofore unrecognized structural features and shows significant differences from the visually derived models.

As described earlier, the most outstanding new feature that emerges from our reconstruction of the 50S subunit is the interface canyon and its associated substructure. None of the visually derived models show this feature, although the latest revision of the Stöffler/Stöffler-Meilicke model has a shallow groove running from the $\mathrm{L} 1$ ridge about half way across the subunit but terminating before the $\mathrm{L} 7 / \mathrm{L} 12$ stalk at a point where in our model the interface canyon is $\sim 4 \mathrm{~nm}$ in both depth and width. The absence of the interface canyon in the visually derived models illustrates the limitations inherent to model building based only on interpretation of a few types of images and without the use of quantitative three-dimensional reconstruction. Other new features of our model are: the three bridges of stain-excluding matter connecting the central protuberance to the main body; the notch at the bottom of the subunit (Figure 4B), which is similar to a feature in the model proposed by Vasiliev et al. (1983); and several smaller knobs and cavities labeled in Figures 4 and 5. Finally, the shape and size of the subunit below the interface canyon (the region labeled 'plateau' in Figure 4A) is quite different in our model from that of the visually derived ones. The significance of some of these features is discussed later.

The models of Stöffler and Stöffler-Meilicke (1984), Lake $(1976,1985)$ and Vasiliev et al. (1983) all show the region of the subunit below the central protuberance to be flat or concave, whereas in the corresponding regions of our model the surface is approximately flat over only a limited area of the surface (labeled 'plateau' in Figure 4A and C) and overall slightly convex. The apparent raison d'etre for the flat or concave surface in the visually derived models is to provide an extended region of complementarity to a part of the interface surface of the corresponding 30S models (Lake, 1976). We propose, based upon the surface topography of the interface side of our model of the $50 \mathrm{~S}$ subunit, that either the interface surfaces of the $50 \mathrm{~S}$ and $30 \mathrm{~S}$ subunits do not interact over as large an area as has been thought, or that current structural models of the $30 \mathrm{~S}$ subunit and $70 \mathrm{~S}$ monosome need to be revised. Certainly the observed modest affinities of the 50S and $30 \mathrm{~S}$ subunits for one another (e.g. Infante et al., 1982) do not require a large area of direct contact between the two in the $70 \mathrm{~S}$ complex.

Three attempts at three-dimensional reconstruction of the 50S subunit have been described (see Introduction), but thus far only one of these has produced a three-dimensional model (Hoppe,

Fig. 4. Three-dimensional model of the 50S ribosomal subunit. Threshold density level the same as was used in Figure 3B. (A) View of the interface surface. (B) Back surface. (C) View rotated by $90^{\circ}$ about vertical axis from (A) or (B). Abbreviations: $\mathrm{CP}$, central protuberance; $\mathrm{BK}$, back knob; $\mathrm{N}$ and $\mathrm{N}^{\prime}$, notch and minor notch; $\mathrm{L} 1$, ridge containing ribosomal protein $\mathrm{L} 1$; P1-P4, pockets 1-4; PL, plateau; R, ridge; ST, stalk containing ribosomal proteins L7 and L12. 

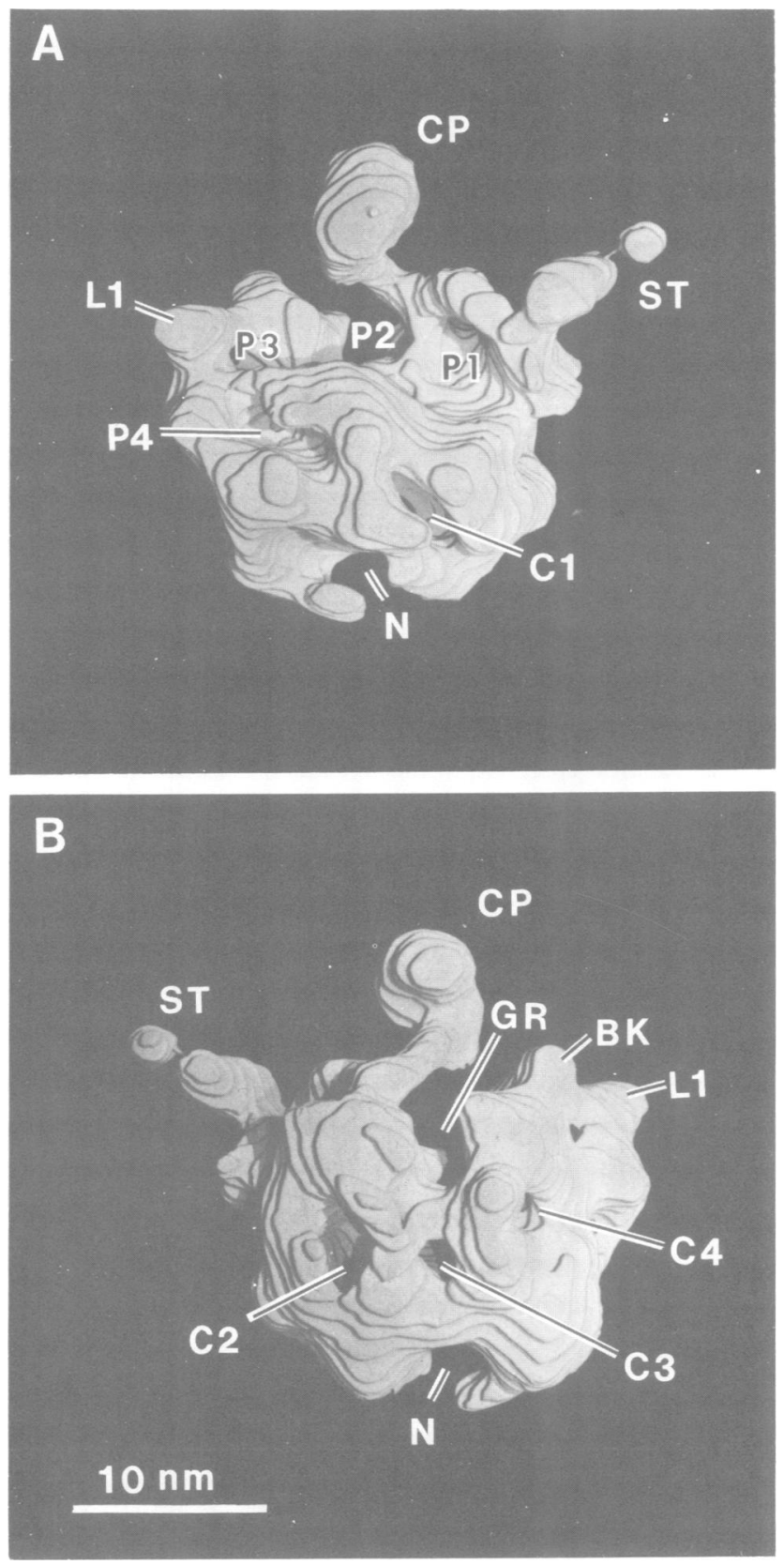

Fig. 5. Three-dimensional model of the $50 \mathrm{~S}$ ribosomal subunit. Same density level as was used to generate surface of Figure 3E. Abbreviations same as in Figure 3 except for the following: $\mathrm{Cl}-\mathrm{C} 4$, cavities 1-4; GR, groove.

1982; Oettl et al., 1983; Hegerl et al., 1984) sufficiently detailed to compare with the model we have obtained. Interestingly, this is the only other model to describe a feature analogous to the interface canyon, although it appears to be smaller than in our model. Unfortunately, Hoppe et al.'s reconstruction of the $50 \mathrm{~S}$ subunit in the crown orientation shows poor contrast near the particle boundaries that are in contact with the upper and lower carbon supporting films. Thus, we can only compare their reconstructions with ours in central sections parallel to the plane of the specimen (e.g. Figure 6). Here the two models appear to agree, except in the region of the central protuberance, where their reconstruction shows some stain penetration that is absent in our model. We believe that our reconstruction is a truer representation of the typical 50S subunit's structure because the electron micrographs were recorded using minimal dose techniques and because data from 490 particles were used to compute the reconstruction, as compared to just one or a few particles in the reconstructions described by Hoppe (1982).

\section{The interface canyon}

In terms of function, this is the most intriguing feature of the three-dimensional model. It is likely to be of importance in protein synthesis because a number of functional sites have been mapped onto this region of the subunit using the technique of immunoelectron microscopy (for reviews see Stöffler and Stöffler-Meilicke, 1984; Lake, 1985).

Based upon immunoelectron microscopic localizations of ribosomal proteins (Lake and Strycharz, 1981) and the binding locations of antibiotics that are known to affect peptidyl transferase activity (Stöffler et al., 1980; Luhrmann et al., 1984; Olson et al., 1982, 1985), the peptidyl transferase active site has been placed near the base of the central protuberance on the side towards the L1 ridge. Two of the pockets forming the interface canyon lie in this region of the three-dimensional model (P2, P3 in Figure 4A). The hole present at the bottom of P2 could provide a means for the growing polypeptide chain to escape from the vicinity of the peptidyl transferase site. Although it would appear from Figure 4A and B that upon emerging from this hole the polypeptide would gain immediate access to the cytoplasm, we do not believe this to be the case (see discussion of exit site below). The binding sites for the elongation factors $\mathrm{G}$ and $\mathrm{Tu}$ have been mapped by immunoelectron microscopy to a region near the base of the stalk (Girshovich et al., 1981, 1986) where, in our model, the deepest of the three pockets (P1) comprising the interface canyon is located. In this region of our model the canyon is 3-4 nm in both breadth and depth. Thus, the interface canyon may contain both the peptidyl transferase center and the sites of binding for the elongation factors.

One consequence of the large size of the interface canyon is that in the 70S monosome there is potentially much more solventaccessible space at the interface between the $30 \mathrm{~S}$ and $50 \mathrm{~S}$ subunits than has been appreciated previously. A number of investigators have tried to correlate all of the available (and sometimes conflicting) biochemical and structural data on the elongation cycle of protein synthesis and have proposed three-dimensional models for the ribosome with tRNA molecules bound at the supposed acceptor and peptidyl sites (Lake, 1977, 1985; Spirin, 1983; Ofengand $e t$ al., 1986). These models could require substantial modification if the peptidyl transferase center is near the interface canyon as we have suggested. Certainly, the additional space provided by the interface canyon allows for orientations and for movements of the tRNA molecules during translocation that previously may have been viewed as unlikely because of steric restrictions.

\section{The exit domain}

The hole present at the base of the middle pocket of the interface canyon (P2 in Figure 4A) probably does not represent directly the site where the nascent polypeptide leaves the ribosome, as such a location would be inconsistent with some known properties of the exit site. First, Bernabeau and Lake (1982) have mapped the exiting nascent polypeptide to a region on the lower half of the back of the 50S subunit by the immunoelectron microscopy technique. Second, experiments on the resistance of the nascent, ribosome-bound polypeptide to proteolysis have shown that $\sim 30$ amino acid residues at the carboxy (polymerizing) end are protected from hydrolysis (Smith et al., 1978). Inspection of the model shown in Figure 5, which was constructed 

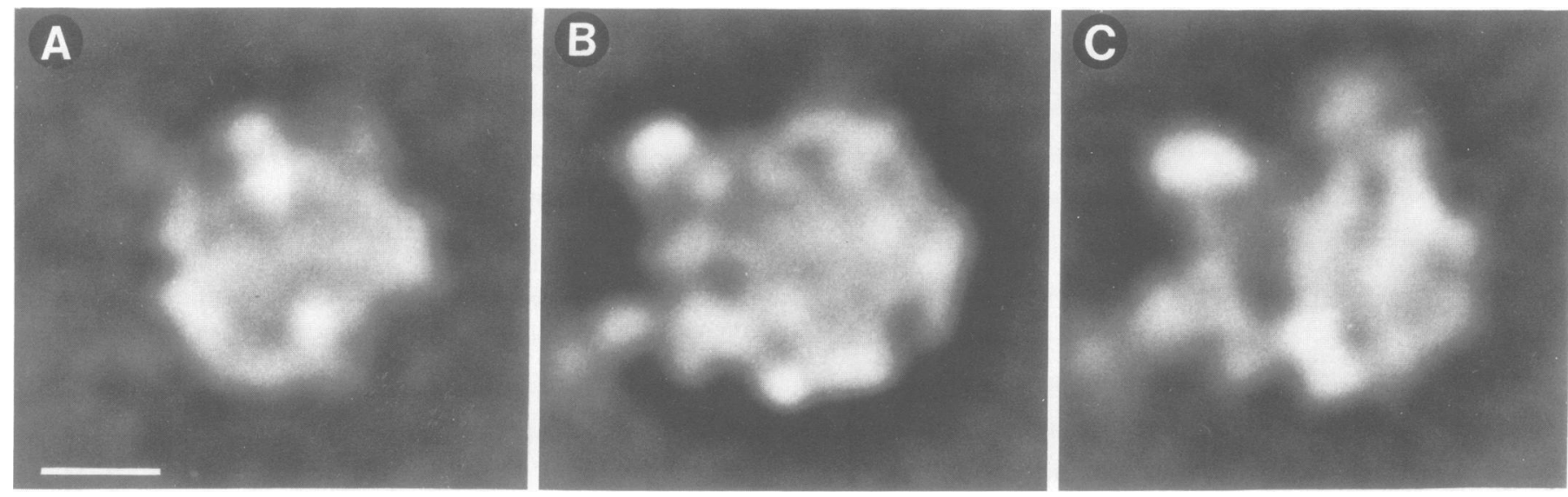

Fig. 6. Sections through the three-dimensional reconstruction: (B) corresponds to a central section; (A) and (C) are taken from a distance $2.5 \mathrm{~nm}$ to either side of (A). Bar represents $10 \mathrm{~nm}$.

by choosing a lower density threshold to represent the surface (see Results) than the level used for the model in Figure 4, reveals a possible pathway for the nascent chain as it emerges from the hole at the base of $\mathrm{P} 2$. From Figure $5 \mathrm{~B}$ it is apparent that some stain penetration occurs around the lower rim of the hole and defines a channel or groove leading down the back of the subunit. It is difficult to determine exactly where this channel emerges at the subunit's surface, but a location about midway down the back of the subunit near cavity C3 (Figure 5B) appears reasonable and would be consistent with the protease protection results; the exit site would then be $\sim 10 \mathrm{~nm}$ from the peptidyl transferase center (assumed to be near P2), a distance which roughly corresponds to the length expected for an extended polypeptide of 30 amino acid residues.

There are at least two other, less defined pathways of stain penetration connecting the interface canyon to the back of the subunit which could also represent the path followed by the exiting polypeptide. One of these connects pocket 3 (Figures 4A and $5 \mathrm{~A}$ ) in the interface canyon to the cavity labeled $\mathrm{C} 4$ (Figure 5B) on the back of the subunit. The other pathway begins in the canyon between pockets 1 and 2 and emerges on the back at cavity C3 (Figure 5B).

\section{Internal structural features}

An intriguing attribute of our three-dimensional reconstruction is that the most strongly stain-excluding regions tend to be located at the periphery of the particle, while significant variations in contrast are present in the internal regions. This can best be seen from the sections through the reconstructed volume presented in Figure 6. Similar behavior was observed in the threedimensional reconstructions described by Hoppe's group (Hoppe, 1982; Oettl et al., 1983; Hegerl et al., 1984), and they attributed it to positive staining of the rRNA. This interpretation is consistent with the neutron and X-ray scattering data which indicates that the ribosomal RNA is located mainly in the central region of the subunit and that the ribosomal proteins are located toward the periphery of the subunit (Stuhrmann et al., 1976; Crichton et al., 1977; Serdyuk et al., 1977, 1979). It is difficult, however, to interpret the internal contrast of our reconstruction solely in terms of positive staining. For example, we do not find any evidence for stain penetrating into the central protuberance, which is known to contain the 5S rRNA (Shatsky et al., 1980a; Evstafieva et al., 1985) and possibly part of the 23S rRNA (Noller and Lake, 1984); instead, the central protuberance is one of the most strongly stain-excluding regions of the subunit (Figure 6; also observ- ed by Selivanova et al., 1986). On the other hand, we do observe a cavity of stain inclusion on the back of the subunit (labeled C2) at the region assigned to the $3^{\prime}$ end of the 23S RNA by immunoelectron microscopy (Shatsky et al., 1980b; StöfflerMeilicke et al., 1981).

Effects other than positive staining could contribute to the small contrast variations observed within the apparent boundaries of the particle. There could, for example, be stain-accessible channels and crevices between the ribosomal proteins and rRNA leading from the surface to the interior regions of the subunit. Also, we cannot exclude the possibility that some of the ribosomal proteins were not present in all of the subunits (Hardy, 1975), which would result in internal regions having densities intermediate between those due to stain and to stain-excluding matter in our reconstruction. Finally, it is possible that some of the internal contrast is due to differences in scattering cross-section between non-stained rRNA and protein (Kühlbrandt, 1982; Kühlbrandt and Unwin, 1982).

\section{The L7/L12 stalk}

In our model the L7/L12 stalk appears to consist of two domains separated by a short narrow bridge of density (Figure 4A and B). This agrees with the model for the L7/L12 dimer proposed by Liljas (1982) in which the L7 and L12 polypeptides (identical except for an acetylated amino terminus in L7) are arranged in a parallel side-by-side manner with the two carboxy terminal domains located at the distal end of the stalk and the two amino terminal portions combining to form a single globular domain which binds to the body of the 50S subuit. The carboxy- and amino-terminal regions are connected by a segment of 13 amino acid residues which is probably flexible. In our reconstruction the carboxy terminal domain is not as large as expected, nor does it appear as two separated subdomains. This is probably because the distal part of the stalk is not accurately represented in our model due to its variability in different images (Verschoor et al., 1985); this causes it to appear blurred in the reconstruction (Figure 6B and C) and smaller than it actually is when the reconstruction is contoured at a density level that is optimal for the other parts of the subunit. There are two copies of the L7/L12 dimer present on average per subunit (Hardy, 1975; Subramanian, 1975), but we cannot yet determine whether the stalk comprises one or both of the dimers. Further study and comparisons of $50 \mathrm{~S}$ subunits containing none, zero and two L7/L12 dimers by the methods described here should resolve this issue. 


\section{Materials and methods}

\section{Specimen preparation and electron microscopy}

Ribosomes were isolated from $E$. coli strain MRE600 obtained as a frozen paste from Grain Processing (Muscatine, IA). The procedure of Staehelin and Maglott (1971) was used. Subunits were obtained by sucrose density gradient centrifugation (10-30\% linear gradients) in a buffer consisting of $10 \mathrm{mM}$ Tris, $100 \mathrm{mM}$ $\mathrm{KCl}, 1 \mathrm{mM} \mathrm{MgCl}, \mathrm{pH}$ 7.5. The subunits were diluted into a buffer consisting of $10 \mathrm{mM}$ Tris $-\mathrm{HCl}, 10 \mathrm{mM} \mathrm{KCl}, 10 \mathrm{mM} \mathrm{MgCl} 2, \mathrm{pH} 7.5$ for electron microscopy.

The procedure described by Stöffler and Stöffler-Meilicke (1984) was used to apply the specimen to grids (300 mesh copper, coated with a thick holey carbon film). With this method a large fraction of the grid surface consists of two layers of carbon with specimen and stain sandwiched between the layers. The negative stain was $0.5 \%$ uranyl acetate, $\mathrm{pH} 4.5$.

A Philips EM420 transmission electron microscope equipped with a low dose kit and goniometer stage was used. Regions of the grid that exhibited uniform negative staining and a suitable distribution of particles were located by scanning at a low magnification and dose rate, or by scanning at a magnification of 49000 taking care not to pre-expose regions to be photographed. Once a suitable area was identified the grid was tilted by $50^{\circ}$, allowed to stabilize for $10-30 \mathrm{~s}$, and photographed with a dose of $<1000 \mathrm{e} / \mathrm{nm}^{2}$ (estimated from the manufacturer's quoted electron speed for Kodak SO163 electron microscope film). The grid was then returned to the untilted state and a second minimal dose micrograph of the same field was recorded. All micrographs were recorded at an instrumental magnification of $49000 \times$ with the microscope operating at $100 \mathrm{kV}$. Only micrographs in which the sandwiched specimen was suspended over holes in the supporting surface were used for further analysis. The micrographs were checked by optical diffractometry for specimen drift, degree of underfocus, and astigmatism.

\section{Image processing}

The three-dimensional reconstruction was calculated with the method developed for three-dimensional reconstruction from a single-exposure, random conical tilt series (Radermacher et al., 1986a). The method will be described in detail elsewhere (Radermacher et al., 1987), and only a brief description is given here. Pairs of micrographs of a large specimen area containing many particles in the crown orientation were recorded, one with the specimen tilted by $50^{\circ}$ and a second without tilt. The direction of the tilt axis in each pair of micrographs was determined by triangulation. Particle coordinates were determined interactively in one micrograph and the corresponding coordinates in the second image of a pair were calculated. After contrast normalization of the complete micrographs the particle images were windowed out, with the sole criterion that the particles showed sufficient separation from each other in the tilted image. The correspondence of the tilted and untilted versions of each particle was established by identical image numbers. The azimuthal angles were determined using a rotational alignment scheme applied to the images windowed out of the untilted micrograph (Frank et al., 1978, 1981; Steinkilberg and Schramm, 1980). A typical crown view was used as a first reference and an average of aligned particles was used as a reference in the following passes. The resulting alignment angles are the azimuthal angles of the images of the tilted particles in the conical tilt series. Images that obviously did not align or showed distorted particles were excluded from further processing after this alignment procedure. With all angles known the images from the tilted micrograph were aligned to a common origin using a cross-correlation scheme analogous to the method described by Guckenberger (1982) (Radermacher et al., 1987). The aligned projections were entered into a three-dimensional reconstruction algorithm designed for arbitrary geometry (Radermacher $e t$ al., 1986a). Three reconstructions were calculated, one using all 489 images and two using half the set of images with 245 and 244 particles each. The latter two were used to determine the reproducible resolution of the reconstruction. Five sections parallel to the specimen near the center of the three-dimensional volume were compared using the $45^{\circ}$ phase residual criterion (Frank et al., 1981). The resolution determined was $3.0-3.2 \mathrm{~nm}$. The Fourier ring correlations were also calculated (Saxton and Baumeister, 1982; van Heel et al., 1982) and yielded a resolution value of $-2 \mathrm{~nm}$. The resolution in the direction of the missing cone (perpendicular to the specimen plane) can be calculated using the elongation factor (Radermacher and Hoppe, 1980), which for a $50^{\circ}$ cone angle is 1.43 , resulting in 4.3 and $2.8 \mathrm{~nm}$ resolution. The reconstruction from all 489 images was low-pass filtered to the resolution of $3.0 \mathrm{~nm}$ and used for the structural interpretation.

\section{Acknowledgements}

This work was supported in part by NIH grant 1R01 GM29169 and NSF grant 8313405 .

\section{References}

Arad,T., Leonard,K., Wittman,H.G. and Yonath,A. (1984) In Csanady,A., Rohlich,P. and Szabo,D. (eds), Electron Microscopy 1984 Vol. 2. Programme Committee of the 8th European Congress on Electron Microscopy, Budapest, pp. $1459-1460$.

Bernabeu,C. and Lake,J.A. (1982) Proc. Natl. Acad. Sci. USA, 79, 3111-3115.

Crichton,R.R., Engelman,D.M., Haas,J., Koch,M.H.J., Moore,P.B., Parfait,R. and Stuhrmann,H.B. (1977) Proc. Natl. Acad. Sci. USA, 74, 5547-5550.

Evstafieva,A.G., Shatsky,I.N., Bogdanov,A.A. and Vasiliev,V.D. (1985) FEBS Lett., 185, 57-62.

Frank,J., Goldfarb,W., Eisenberg,D. and Baker,T.S. (1978) Ultramicroscopy, $3,283-290$.

Frank,J., Verschoor,A. and Boublik,M. (1981) Science, 214, 1353-1355.

Frank,J., Verschoor,A. and Wagenknecht,T. (1985) In Wu,T.T. (ed.), New Methodologies in Studies of Protein Configuration. van Nostrand Reinhold, New York, pp. 36-89.

Girshovich,A.S., Kurtskhalia,T.V., Ovchinnikov, Yu,A. and Vasiliev,V.D. (1981) FEBS Lett., 130, 54-59.

Girshovich,A.S., Bochkareva,E.S. and Vasiliev,V.D. (1986) FEBS Lett., 197, $192-198$.

Guckenberger,R. (1982) Ultramicroscopy, 9, 167-174.

Hardy,S.J.S. (1975) Mol. Gen. Genet., 140, 253-274.

Hegerl,R., Hoppe,W., Knauer,V. and Typke,D. (1984) In Csanady,A., Röhlich,P. and Szabo,D. (eds), Electron Microscopy 1984. Programme Committee of the 8th European Congress on Electron Microscopy, Budapest, pp. 1363-1372.

Hoppe,W. (1982) Electron Microscopy 1982. Proc. 10th Intl. Congr. Deutsche Gesellschaft für Elektronenmikroskopie, Hamburg, Vol. 1, pp. 97-106.

Hoppe,W. (1983) Angew. Chem., 95, 465-494 (Int. Ed. Engl., 22, 456-485). Infante,A.A., Demple,B. and Chaires,J.B. (1982) J. Biol. Chem., 257, 80-87.

Kiselev,N.A., Orlova,E.V., Stel'mashchuk,V. Ya., Vasiliev,V.D., Selivanova,O.M., Kosykh,V.P., Pustovskikh,A.I. and Kirichuk,V.S. (1983) J. Mol. Biol., 169, 345-350.

Kühlbrandt,W. (1982) Ultramicroscopy, 7, 221-232.

Kühlbrandt,W. and Unwin,P.N.T. (1982) J. Mol. Biol., 156, 431-448.

Lake,J.A. (1976) J. Mol. Biol., 105, 131-159.

Lake,J.A. (1977) Proc. Natl. Acad. Sci. USA, 74, 1903-1907.

Lake,J.A. (1985) Annu. Rev. Biochem., 54, 507-530.

Lake,J.A. and Strycharz,W.A. (1981) J. Mol. Biol., 153, 979-992.

Liljas,A. (1982) Prog. Biophys. Mol. Biol., 40, 161-228.

Luhrmann,R., Bald,R., Stöffler-Meilicke,M. and Stöffler,G. (1984) Proc. Natl. Acad. Sci. USA, 78, 7276-7280.

Meisenberger,O., Pilz,I., Stöffler-Meilicke,M. and Stöffler,G. (1984) Biochim. Biophys. Acta., 781, 225-233.

Noller,H.F. and Lake,J.A. (1984) In Bittar,E. (ed.), Membrane Structure and Function. Wiley, New York, Vol. 6, pp. 217-297.

Oettl,H., Hegerl,R. and Hoppe,W. (1983) J. Mol. Biol., 163, 431-450.

Ofengand,J., Ciesiolka,J., Dennman,R. and Nurse,K. (1986) In Hardesty,B. and Kramer,G. (eds), Structure, Function and Genetics of Ribosomes. Springer, New York, in press.

Olson,H.M., Grant,P.G., Cooperman,B.S. and Glitz,D.G. (1982) J. Biol. Chem., 257, 2649-2656.

Olson,H.M., Nicholson,A.W., Cooperman,B.S. and Glitz,D.G. (1985) J. Biol. Chem., 260, 10326-10331.

Radermacher,M. and Hoppe,W. (1980) In Brederoo,P. and de Priester,W. (eds), Electron Microscopy 1980. 7th Eur. Congr. Electron Microsco. Found., Leiden, 1, 132-133.

Radermacher,M., Wagenknecht,T., Verschoor,A. and Frank,J. (1986a) $J$. Microsc., 141, RP1-RP2.

Radermacher,M., Wagenknecht,T., Verschoor,A. and Frank,J. (1986b) In Bailey,G.W. (ed.), Proc. 44st Annu. Meeting EMSA, San Francisco Press, San Francisco, 40-41.

Radermacher,M., Wagenknecht,T., Verschoor,A., Frank,J. (1987) J. Microsc., in press.

Saxton,W.O. and Baumeister,W. (1982) J. Microsc., 127, 127-138.

Selivanova,O.M., Gongadze,G.M., Gudkov,A.T. and Vasiliev,V.D. (1986) FEBS Lett., 197, 79-83.

Serdyuk,I.N., Grenader,A.K. and Koteliansky,V.E. (1977) Eur. J. Biochem., 79, 504-508.

Serdyuk,I.N., Grenader,A.K. and Zaccai,G. (1979) J. Mol. Biol., 135, 691-707.

Shatsky,I.N., Evstafieva,A.G., Bystrova,T.F., Bogdanov,A.A. and Vasiliev,V.D. (1980a) FEBS Lett., 121, 97-100.

Shatsky.I.N., Evstafieva,A.G., Bystrova,T.F., Bogdanov,A.A. and Vasiliev,V.D. (1980b) FEBS Lett., 122, 251-255. 
Smith,W.P., Tai,P.-C, and Davis,B.D. (1978) Proc. Natl. Acad. Sci. USA, 75, 5922-5925.

Staehelin,T. and Maglott,D.R. (1971) Methods Enzymol., 20, 449-456.

Steinkilberg,M. and Schramm,J. (1980) Hoppe-Seyler's Z. Physiol. Chem., 361, $1363-1369$.

Spirin,A.S. (1983) FEBS Lett., 156, 217-221.

Stöffler,G. and Stöffler-Meilicke,M. (1983) In Tschesche,H. (ed), Modern Methods in Protein Chemistry. de Gruyter, New York, pp. 409-457.

Stöffler,G. and Stöffler-Meilicke,M. (1984) Annu. Rev. Biophys. Bioeng., 13, $303-330$.

Stöffler,G., Bald,R., Kastner,B., Luhrmann,R., Stöffler-Mielicke,M. and Tischendorf,G. (1980) In Chambliss,G., Craven,G.R., Davies,J., Davis,K., Kahan,L. and Nomura,M. (eds), Ribosome: Structure, Function and Genetics. University Park Press, Baltimore, pp. 171-205.

Stöffler-Meilicke,M., Noah,M. and Stöffler,G. (1983) Proc. Natl. Acad. Sci. USA, 80, 6780-6784.

Stöffler-Meilicke,M., Stöffler,G., Odom,O.W., Zinn,A., Kramer,G. and Hardesty,B. (1981) Proc. Natl. Acad. Sci. USA, 78, 5538-5542.

Stuhrmann,H.B., Haas,J., Ibel,K., DeWolf,B., Koch,M.H.J., Parfait,R. and Crichton,R.R. (1976) Proc. Natl. Acad. Sci. USA, 73, 2379-2383.

Subramanian,A.R. (1975) J. Mol. Biol., 95, 1.

Tardieu,A. and Vachette,P. (1982) EMBO J., 1, 35-40.

Tischendorf,G.W., Zeichhardt,H. and Stöffler,G. (1974) Mol. Gen. Genet., 134, 187-208.

van Heel,M., Keegstra,W., Schutter,W. and van Bruggen,E.F.J. (1982) In Wood,E.J. (ed.), Life Chemistry Reports, Supplement 1, The Structure and Function of Invertebrate Respiratory Proteins. EMBO Workshop, Leeds, pp. 69-73.

Vasiliev,V.D., Selivanova,O.M., Baranov,V.I. and Spirin,A.S. (1983) FEBS Lett., 155, 167-172.

Verschoor,A., Frank,J. and Boublik,M. (1985) J. Ultrastruct. Res., 92, 180-189.

Wittman,H.G. (1983) Annu. Rev. Biochem., 52, 35-65.

Yonath,A. (1984) Trends Biochem. Sci., 9, 227-230.

Received on October 3, 1986; revised on January 12, 1987 